\title{
QUANTIFICAÇÃO DO TEOR DE BIODIESEL DE CRAMBE EM MISTURAS COM DIESEL UTILIZANDO ESPECTROSCOPIA MIR E SELEÇÃO DE VARIÁVEIS
}

\author{
Lucas G. da Costa ${ }^{\mathrm{a}, *,(\mathbb{D})}$, Baltazar V. Sitoe ${ }^{\mathrm{a}, \mathrm{b}}$, Douglas Q. Santos ${ }^{\mathrm{c}}$ e Waldomiro Borges Neto ${ }^{\mathrm{a}}$ \\ anstituto de Química, Universidade Federal de Uberlândia, 38408-100 Uberlândia - MG, Brasil \\ ${ }^{b}$ Faculdade de Ciências Naturais e Matemática, Universidade Púnguè, 323 Chimoio - Manica, Moçambique \\ 'Escola Técnica de Saúde, Universidade Federal de Uberlândia, 38408-902 Uberlândia - MG, Brasil
}

Recebido em 14/01/2020; aceito em 03/03/2020; publicado na web em 25/05/2020

\begin{abstract}
QUANTIFICATION OF CRAMBE BIODIESEL CONTENT IN DIESEL BLENDS USING MIR SPECTROSCOPY END VARIABLES SELECTION. Mid-Infrared absorption spectroscopy associate with the Partial Least Squares regression is the official method to monitor Brazilian commercial diesel quality. This method, however, uses solvents and a large number of samples for the construction of the calibration curves, which generates waste and increases the time needed for the analysis. In order to develop a non-destructive method, being possible to recover the sample after its quantification, decrease the quantity of samples and make use of a single calibration curve, in this study we used the oilseed crambe, which is not used in human food, for the methyl biodiesel production and PLS analysis for their content determination in mixtures with diesel at a concentration range of 1.00 to $30.00(\% \mathrm{v} / \mathrm{v})$. The global model for crambe methyl biodiesel obatined RMSEC $=0.26(\% \mathrm{v} / \mathrm{v}), \mathrm{RMSECV}=0.35(\% \mathrm{v} / \mathrm{v})$ and RMSEP $=0.41(\%$ $\mathrm{v} / \mathrm{v})$. Complementary, variable selection method iPLS was applied in the global model in order to reduce the spectral range required to regression construction and to improve the RMSEP, RMSECV and RMSEC values.
\end{abstract}

Keywords: biofuels; multivariate calibration; selection of Intervals; iPLS.

\section{INTRODUÇÃo}

O aumento exponencial da população mundial e os avanços tecnológicos demandam cada vez mais, fontes de energia que sejam preferencialmente renováveis e que seus usos como combustíveis não colidam com outros de grande significância, como o uso na alimentação. ${ }^{1} \mathrm{O}$ desenvolvimento de processos sustentáveis que convertam biomassa em produtos com valor agregado contribui de maneira efetiva no aproveitamento de resíduos, geração de renda e redução dos impactos negativos ao meio ambiente. ${ }^{2}$

\section{Biodiesel}

Biodiesel é o combustível feito a partir de óleo vegetal, residual ou gordura animal, e álcool anidro na presença de um catalisador. Os óleos e gorduras são compostos por carbono, hidrogênio e oxigênio que se assemelham estruturalmente quanto à presença de triacilglicerídeos (TAG) de ácidos graxos, mas que se diferenciam pelo tipo e disposição destes ácidos quando combinados com o glicerol. ${ }^{3}$

O processo mais utilizado para a obtenção do biodiesel é a transesterificação homogênea via catálise básica. Essa reação consiste na interação entre os TAG constituintes dos óleos vegetais/gorduras animais e um álcool de cadeia curta na presença de uma base de Brönsted (receptora de prótons) que atua como catalisador, gerando o éster (chamado de biodiesel) e glicerol. ${ }^{4}$

Apesar de a transesterificação ser uma reação simples, muitas variáveis podem interferir no processo, como a relação molar dos reagentes e condição inicial do óleo utilizado. O teor elevado de ácidos graxos livres (AGL) contidos em determinados óleos vegetais pode influenciar qualitativa e quantitativamente na transesterificação, sendo que matérias-primas contendo acidez maior que $0,50(\% \mathrm{~m} / \mathrm{m})$ de AGL e umidade maior que $0,25(\% \mathrm{~m} / \mathrm{m})$ de água não são adequadas para a reação, fazendo-se necessário o emprego prévio de uma reação de

*e-mail: lucasgustavodacosta@gmail.com esterificação (ou hidroesterificação) para reduzir o número de AGL presentes no óleo. ${ }^{5}$

A soja é a oleaginosa mais utilizada para produção do biodiesel no Brasil por se tratar de uma cultura consolidada, com uma maquinaria bem desenvolvida para o plantio, colheita, extração e refino de seu óleo, porém este mesmo óleo possui alto valor agregado por ter como uso principal, a alimentação humana. ${ }^{6}$ Em contra partida, o crambe (Crambe abyssinica Hochst) é uma oleaginosa nativa do continente africano que apresenta grande adaptabilidade ao solo e clima brasileiro. É uma planta resistente à geadas e a seca, tem produtividade entre 1000 e $1500 \mathrm{Kg} / \mathrm{ha}$, com sementes possuindo por volta de 35,00 a 60,00 (\% m/m) de óleo. Sua cultura é considerada de ciclo curto (média de 90 dias), sendo uma boa opção para a safrinha por se tratar de uma cultura de outono/ inverno. Para a extração do óleo, tradicionalmente é usada a prensagem e/ou extração com solventes orgânicos, resultando em um produto com grande teor de AGL, principalmente o ácido úrico (cerca de 60,00 (\% v/v)), que é altamente tóxico e inviabiliza o uso como alimento, ${ }^{7-9}$ mas com o tratamento adequado por meio da hidroesterificação, se torna um bom substituinte do óleo de soja na produção de biodiesel. ${ }^{8}$

\section{Controle de Qualidade}

O diesel combustível vendido no território brasileiro é composto de biodiesel (principalmente feito a partir de soja e gordura animal) e diesel, em proporção evolutiva até se atingir o valor de $15,00 \pm 0,50(\% \mathrm{v} / \mathrm{v})$ biodiesel-diesel em maio de 2023. ${ }^{10-12}$ Segundo estudo publicado pela Agência Nacional do Petróleo, Gás Natural e Biocombustíveis (ANP), 50\% de não conformidades encontradas em diesel comercial estão relacionadas a essa quantidade de biodiesel na mistura. ${ }^{13} \mathrm{O}$ alto custo dos biodieseis e a dificuldade de se verificar visualmente misturas biodiesel-diesel não conformes incentivam a adulteração criminosa, ou ainda, os meios de transporte utilizados na distribuição do combustível podem propiciar contaminações por outros produtos petrolíferos. As adulterações e contaminações nas 
misturas alteram suas composições, podendo causar danos aos veículos. ${ }^{14,15}$ e diminuir as vantagens ambientais esperadas.

A norma NBR-15568 é o método oficial empregado na determinação do teor de biodiesel em diesel no Brasil, utilizando a Espectroscopia no Infravermelho Médio e a calibração multivariada por Quadrados Mínimos Parciais (do inglês, $P L S$ ). Neste método, duas curvas de calibração são construídas, uma com 160 pontos (amostras), e outra com 45 , nas faixas de concentração de 0,00 a 8,00 (\% v/v) para a primeira e de 8,00 a 30,00 (\% v/v) para a segunda. Há a necessidade de utilizar solventes como o iso-octano e o hexano na preparação das mostras e quando o modelo se mostra inadequado para as predições (com erros elevados), a adição de grupos de 20 amostras é necessária. ${ }^{16}$

Visando reduzir o tempo necessário para a análise do teor dos biodieseis em misturas com diesel e diminuir a geração de resíduos após a leitura das amostras, este trabalho tem como intuito o desenvolvimento de um método para a quantificação de biodiesel metílico de crambe presente em misturas com diesel que se diferencie e apresente vantagens em relação ao método oficial como: não usar solventes na preparação das amostras, construir uma única curva de calibração contemplando a mesma faixa das duas curvas do método oficial e diminuir a quantidade de pontos usados na construção do modelo.

\section{Quimiometria}

A quimiometria é a parte da química que se utiliza de ferramentas estatísticas, matemáticas e de lógica formal para obter o máximo de informações a partir da análise de dados químicos. Os avanços na instrumentação analítica, gerando dados com muitas variáveis e o grande uso dessas medidas necessita de métodos de estatística multivariada, álgebra matricial e análise numérica para converter a resposta instrumental no dado químico de interesse, ${ }^{17,18}$ assim, a quimiometria se firma como uma importante parte da química analítica.

\section{Quadrados Mínimos Parciais}

A regressão por Quadrados Mínimos Parciais é o método mais usado para a construção de modelos de calibração multivariada por não ser necessário um conhecimento exato de todos os componentes presentes em uma amostra, realizando a previsão mesmo em presença de interferentes, contanto que esses interferentes também estejam presentes na construção do modelo. ${ }^{19}$

No modelo $P L S 1$, uma matriz $\mathbf{X}$ (correspondente ao sinal instrumental das amostras) e um vetor $\mathbf{y}$ (correspondente às concentrações do analito de interesse) são incorporados e um processo iterativo aperfeiçoa ao mesmo tempo a projeção das amostras (X) sobre os pesos (y) determinando assim os escores (Variáveis Latentes), que são ajustados por uma função linear minimizando os desvios. Essa otimização simultânea provoca pequenas mudanças nas direções dos pesos, os fazendo perder a ortogonalidade e levando a redundâncias que evidenciam a relação linear entre eles. ${ }^{20}$

Dois grupos de amostras são usados na construção do modelo: o grupo de calibração, que estabelece a relação entre a resposta instrumental e a propriedade de interesse, criando a equação matemática responsável pela regressão (ou seja, é o modelo em si) e o grupo de previsão, usado para testar o modelo. Para a determinação correta do número de Variáveis Latentes (VL) do modelo, se usa o método de validação cruzada, que consiste no uso de amostras do grupo de calibração para testar a capacidade preditiva do modelo antes do grupo de previsão. ${ }^{21,22}$

Com o intuito de produzir modelos de regressão com melhor desempenho e menores erros de previsão, algoritmos determinísticos têm sido utilizados para a seleção de variáveis com maior relevância de informação para a quantificação de determinado analito, localizando regiões no espectro com maior variância entre as amostras. ${ }^{23,24}$

\section{Quadrados Mínimos Parciais por Intervalos}

O iPLS (PLS por intervalos) divide o espectro em subintervalos de igual tamanho e calcula um modelo $P L S$ para cada uma dessas faixas. Estes submodelos são comparados com o modelo global, geralmente pelo parâmetro de Erro Quadrático Médio de Validação Cruzada (do inglês, RMSECV), assim as regiões com parâmetros de validação melhores que o modelo global são selecionadas para a construção de um novo modelo $P L S .^{23}$

\section{Validação}

A validação é um processo de verificação que certifica se um método desenvolvido apresenta um desempenho adequado nas condições em que ele será aplicado e se dá através da determinação de diversos parâmetros conhecidos como figuras de mérito. Em modelos multivariados, recomenda-se que as figuras de mérito sejam calculadas através do Sinal Analítico Líquido (do inglês NAS). ${ }^{24-26}$

O NAS, é a parte do sinal analítico ortogonal às contribuições de outros possíveis constituintes da amostra e com seu uso é possível calcular um valor escalar livre de interferentes. Assim, o modelo multivariado pode ser representado em uma forma pseudo-univariada. ${ }^{27}$ Para o cálculo do NAS se usa a matriz de dados reconstruída com $A$ variáveis latentes, $\left(\hat{\mathbf{X}}_{\mathrm{A}}\right)$, determinando em seguida a matriz que contém a informação de todas as espécies presentes na amostra, exceto da espécie de interesse $K$, Equação 1, isso faz com que a matriz $\hat{\mathbf{X}}_{\mathrm{A},-\mathrm{K}}$ fique livre de qualquer contribuição da espécie $K$. Logo, o vetor $\boldsymbol{N A S}$ é obtido conforme a Equação 2:

$$
\begin{gathered}
\hat{\mathbf{X}}_{\mathrm{A},-\mathrm{K}}=\left[\mathbf{I}-\hat{\mathbf{y}}_{\mathrm{A}, \mathrm{K}} \hat{\mathbf{y}}_{\mathrm{A}, \mathrm{K}}^{+}\right] \hat{\mathbf{X}}_{\mathrm{A}} \\
\hat{\mathbf{x}}_{\mathrm{A}, \mathrm{K}}^{\mathrm{nas}}=\left[\mathbf{I}-\hat{\mathbf{X}}_{\mathrm{A},-\mathrm{K}}^{\mathrm{T}}\left(\hat{\mathbf{X}}_{\mathrm{A},-\mathrm{K}}^{\mathrm{T}}\right)^{+}\right] \hat{\mathbf{X}}_{\mathrm{A}}
\end{gathered}
$$

onde $\hat{\mathbf{X}}_{\mathrm{A},-\mathrm{K}}$ é uma matriz com todas as informações exceto a espécie de interesse, $\boldsymbol{I}$ é a matriz identidade, $\hat{\mathbf{y}}_{\mathrm{A}, \mathrm{K}}$ é o vetor de concentrações da espécie de interesse $K$ estimado com $A$ variáveis latentes, o índice "+" sobrescrito indica a pseudo-inversa do vetor, $\hat{\mathbf{X}}_{\mathrm{A}}$, a matriz de dados reconstruída com $A$ variáveis latentes através do algoritmo SIMPLS e $\hat{\mathbf{x}}_{\mathrm{A}, \mathrm{K}}^{\text {nas }}$ é o vetor $\boldsymbol{N A S}$.

O Erro Quadrático Médio (do inglês RMSE) fornece informações sobre o ajuste do modelo em relação aos dados de cada grupo, de calibração (do inglês $R M S E C$ ), validação cruzada (do inglês $R M S E C V$ ) e previsão (do inglês $R M S E P$ ) ${ }^{28}$ obtidos através da Equação 3:

$$
\operatorname{RMSE}=\sqrt{\frac{\sum_{\mathrm{i}=1}^{\mathrm{n}_{\mathrm{g}}}\left(\mathbf{y}_{\mathrm{p}}-\mathbf{y}_{\mathrm{r}}\right)^{2}}{\mathrm{n}_{\mathrm{g}}}}
$$

em que $\mathbf{y}_{\mathrm{p}}$ é o valor de concentração da espécie de interesse previsto, $\mathbf{y}_{\mathrm{r}}$ o valor real de concentração da espécie de interesse presente na amostra e $\mathrm{n}_{\mathrm{g}}$ o número de amostras presentes em cada grupo.

A Sensibilidade (SÊN), por definição é a fração do sinal responsável pelo acréscimo de uma unidade de concentração à propriedade de interesse ${ }^{26}$ e pode ser calculada através da Equação 4:

$$
\mathrm{SENN}=\mathbf{s}_{\mathrm{K}}^{\text {nas }}
$$

onde $\mathbf{s}_{\mathrm{K}}^{\text {nas }}$ é o vetor sensibilidade líquida, obtido través da Equação 5:

$$
\mathbf{s}_{\mathrm{K}}^{\mathrm{nas}}=\frac{\hat{\mathbf{x}}_{\mathrm{A}, \mathrm{K}}^{\mathrm{nas}}}{\mathbf{y}}
$$

em que $\hat{\mathbf{x}}_{\mathrm{A}, \mathrm{K}}^{\text {nas }}$ é o vetor $\boldsymbol{N A S}$ calculado pela Equação 2 e $\boldsymbol{y}$ é o vetor concentração.

A Sensibilidade Analítica, Equação 6, é a razão entre a 
Sensibilidade e o desvio padrão do sinal de referência: ${ }^{26}$

$$
\gamma=\frac{\mathrm{SÊN}}{\delta \mathrm{x}}
$$

onde $\gamma$ é a Sensibilidade Analítica, SÊN é a Sensibilidade calculada pela Equação 4 e $\delta$ x é o desvio padrão do sinal estimado pelo $N A S$ para 15 espectros do sinal de referência. O inverso desta figura de mérito $\left(\gamma^{1}\right)$ estabelece a menor diferença de concentração entre amostras distinguível pelo modelo..$^{29}$

Os Limites de Detecção e Quantificação, Equações 7 e 8, expressam as menores quantidades da espécie de interesse que pode ser detectada e quantificada, respectivamente. ${ }^{28}$

$$
\begin{aligned}
& \mathrm{LD}=3 \delta \mathrm{x} \frac{1}{\mathrm{SENN}} \\
& \mathrm{LQ}=10 \delta \times \frac{1}{\mathrm{SÊN}}
\end{aligned}
$$

Em que $\delta x$ é o desvio padrão do sinal de referência e SÊN é obtido pela Equação 4.

\section{Comparação entre modelos multivariados}

Modelos gerados por análises multivariadas podem ser comparados se utilizado dos valores de $R M S E P$ gerados e a distribuição de Fisher-Snedecor (teste F) de acordo com a Equação 9:

$$
F_{\text {cal }}=\left(\frac{\text { RMSEP }_{1}}{\operatorname{RMSEP}_{2}}\right)^{2}
$$

onde $R M S E P_{1}$ é maior que $R M S E P_{2}$. Se o valor de $\mathrm{F}_{\text {cal }}$ for maior que o valor tabelado da distribuição de Fisher-Snedecor com graus de liberdade igual ao número de amostras de predição e um nível de significância de 5\%, não há evidência estatística de que os valores seguem a distribuição normal então, o modelo com $R M S E P_{2}$ apresenta melhor exatidão. ${ }^{30}$

\section{PARTE EXPERIMENTAL}

\section{Síntese do Biodiesel}

O óleo de crambe passou por um processo de hidroesterificação na proporção de massas $1: 1(\mathrm{~m} / \mathrm{m})$ óleo/álcool metílico mais $0,5(\% \mathrm{~m} / \mathrm{m})$ de $\mathrm{H}_{2} \mathrm{SO}_{4}$ em relação ao volume de álcool, em fervura por $120 \mathrm{~min}$ sob refluxo. Em seguida, se separou a fase óleo e a ela, foi adicionado álcool metílico na razão de $30 \%$ de sua massa mais $1(\% \mathrm{~m} / \mathrm{m})$ de $\mathrm{KOH}$. A mistura foi deixada em agitação por $60 \mathrm{~min}$ e depois transferida para um funil de separação para descanso de $24 \mathrm{~h}$. Depois do período de descanso, a fase glicerol da catálise básica foi retirada e o éster (biodiesel), lavado com três porções de água destilada a $80{ }^{\circ} \mathrm{C}$. $\mathrm{O}$ éster foi levado a um evaporador rotativo por $60 \mathrm{~min}$, a $80 \mathrm{rpm}$, pressão de $400 \mathrm{mmHg}$ e $80^{\circ} \mathrm{C}$ para a retirada de possíveis traços de água.

\section{Preparação das amostras}

As amostras foram construídas usando diesel puro, cedido pela TRANSPETRO S/A, localizada em Uberlândia - MG e o biodiesel preparado no Laboratório de Quimiometria do Triângulo, do Instituto de Química da Universidade Federal de Uberlândia, na faixa de 1,00 a 30,00 (\% v/v) biodiesel-diesel, sendo 43 amostras para o conjunto denominado calibração e 25 para o conjunto de previsão (totalizando 68).
A quantidade de amostras foi determinada seguindo o recomendado pela norma da American Society for Testing and Materials, ASTM E1655-05, ${ }^{11}$ que apresenta as Equações 10 e 11, como método de calcular a quantidade mínima de amostras para a construção de uma curva de calibração usando o $P L S$, de acordo com a quantidade de VL que se deseja obter no modelo.

$$
\begin{gathered}
\mathrm{C}_{\mathrm{p}}=6(\mathrm{k}+1) \\
\mathrm{C}_{\mathrm{v}}=4 \mathrm{k}
\end{gathered}
$$

onde $\mathrm{C}_{\mathrm{p}}$ é a quantidade de amostras do conjunto de calibração, $k$ é o número de $\mathbf{V L}$ que se deseja obter no modelo e $\mathrm{C}_{\mathrm{v}}$ é a quantidade de amostras do conjunto validação. Quando $k \leq 3$, o grupo de calibração deverá ter no mínimo, 24 amostras.

\section{Obtenção e processamento dos espectros MIR}

Os espectros de absorbância dos dois conjuntos da mistura biodiesel de crambe-diesel foram obtidos em quintuplicata de leitura, na região de 4000 a $600 \mathrm{~cm}^{-1}$, no equipamento PerkinElmer - Spectrum Two, utilizando o Acessório Horizontal de Refletância Total Atenuada (do inglês, HATR) com cristal de ZnSe, resolução de $4 \mathrm{~cm}^{-1}$ e 16 varreduras.

Os dados espectrais foram transferidos para ambiente MATLAB, versão R2015a, tiveram suas linhas de base corrigidas, calculada a média das replicatas de cada amostra e a matriz final usada na regressão foi obtida depois de um corte na região de interesse espectral ficando com o tamanho de 68 amostras por 2925 variáveis conforme a Figura 1.

O modelo global foi construído usando o PLS_Toolbox, versão 8.61 , tendo como pré-processamento o mean center em ambos os blocos (X, amostras, e $\mathbf{y}$, vetor concentração) e vanetian blinds cross-validation como método de validação cruzada (12 janelas com 2 amostras por janela). Os modelos $i P L S$ foram obtidos através do iPLS_Toolbox para 5, 10, 15, 20, 25, 30, 35 e 40 faixas, sendo posteriormente recalculados usando o PLS_Toolbox 8.61.

\section{RESULTADOS E DISCUSSÃO}

\section{Modelo global}

A quantidade de variáveis latentes é determinada de modo a alcançar o menor valor de RMSECV e a melhor variância explicada, portanto foram escolhidas 4 VL para a construção do modelo global (que contém todas as variáveis) apresentado na Figura 2 e que obteve $R M S E C=0,26(\% \mathrm{v} / \mathrm{v}), R M S E C V=0,35(\% \mathrm{v} / \mathrm{v}), R M S E P=0,41$ $(\% \mathrm{v} / \mathrm{v})$, varianciância de $99,23 \%$ na matriz $\mathbf{X}$ de sinais instrumentais e $99,89 \%$ no vetor $\mathbf{y}$ de concentrações.

$\mathrm{O}$ valor de $R M S E C$ menor que o RMSEP, nos diz que o modelo não possui tendência em suas previsões e através do coeficiente de correlação (R) é possível perceber ainda, uma boa relação entre os dados de referência e os calculados pelo modelo, já que quanto mais próximo de 1, melhor é a concordância entre os valores medidos e previstos.

Os dados referentes à Sensibilidade, Sensibilidade Analítica, Inverso da Sensibilidade Analítica, LQ e LD expostos na Tabela 1, obtiveram valores satisfatórios. Os limites de Detecção e Quantificação menores que o primeiro ponto da curva reforçam a empregabilidade da faixa de trabalho escolhida, que contempla a evolução do percentual de biodiesel prevista pela legislação, evitando assim a necessidade de construção de novos modelos a cada mudança de concentração mínima exigida de biodiesel em diesel comercial. 


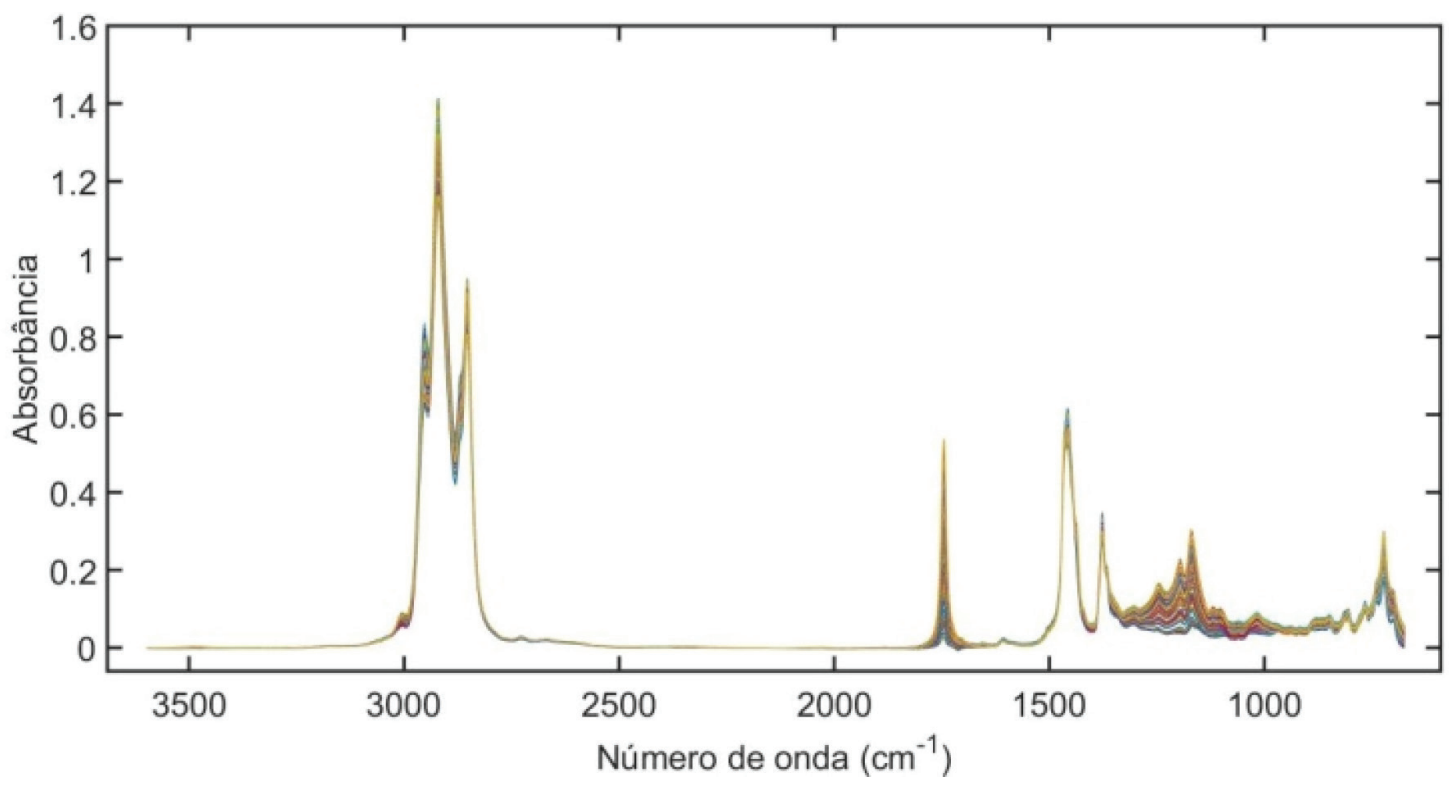

Figura 1. Espectros de absorbância da mistura biodiesel metílico de crambe-diesel depois de corrigidos

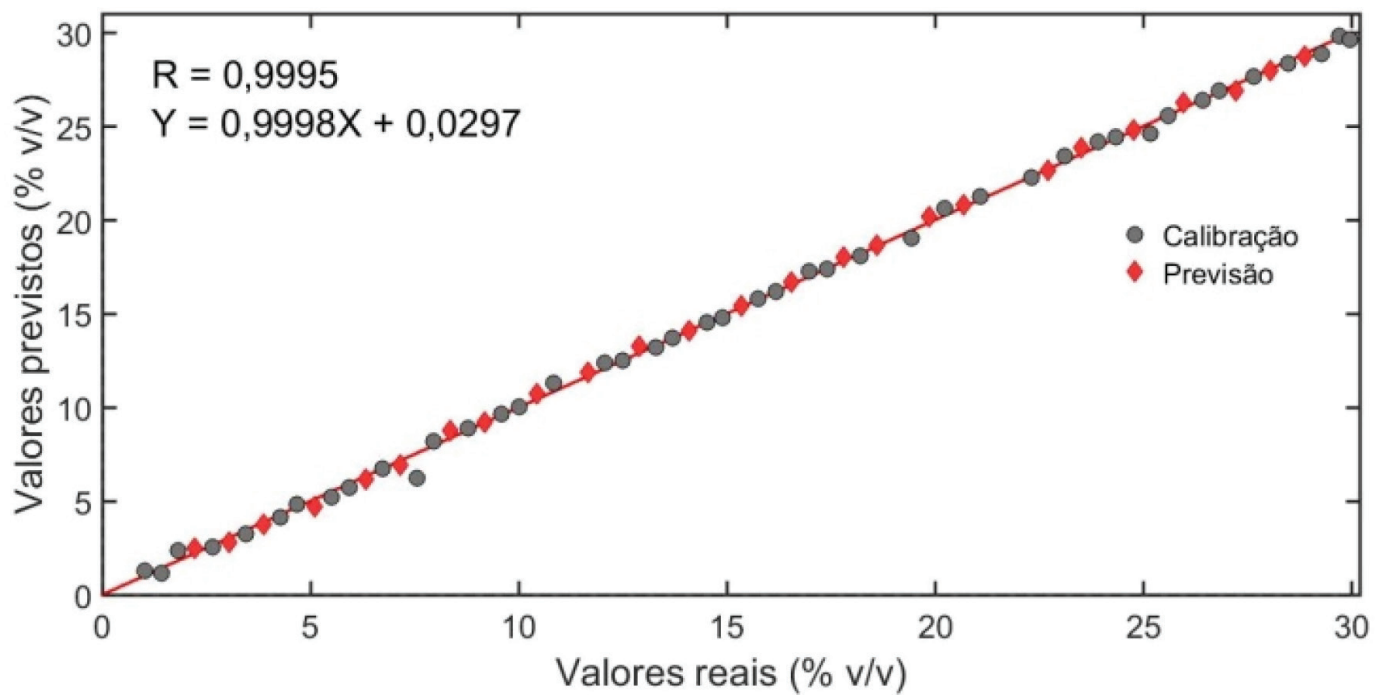

Figura 2. Modelo global de regressão por PLS

Tabela 1. Figuras de mérito para o modelo global biodiesel metílico de crambe-diesel

\begin{tabular}{ll}
\hline Sensibilidade (\% v/v) & 0,07 \\
Sensibilidade Analítica (\% v/v) & 27,0 \\
Inverso da Sensibilidade Analítica (\% v/v) & 0,04 \\
LQ (\% v/v) & 0,13 \\
LD (\% v/v) & 0,04 \\
\hline
\end{tabular}

\section{Modelo gerado por $i P L S$}

Utilizando a segunda faixa obtida quando se dividiu a região espectral em quarenta intervalos (variáveis de 75:148) destacada na Figura 3 e que corresponde às vibrações relacionadas às deformações angulares de $\mathrm{CH}_{2}$ de alcenos, foi calculado o novo modelo $P L S$ (iPLS40) empregando como pré-processamento o mean center em ambos os blocos e vanetian blinds cross validation como método de validação cruzada.

A Figura 4 apresenta a regressão construída após o uso do iPLS. Com 4 VL, RMSEC $=0,23(\% \mathrm{v} / \mathrm{v}), R M S E C V=0,38(\% \mathrm{v} / \mathrm{v})$,
RMSEP $=0,24(\% \mathrm{v} / \mathrm{v})$, varianciância de $99,91 \%$ na matriz $\mathbf{X}$ de sinais instrumentais e $99,92 \%$ no vetor $\mathbf{y}$ de concentrações, este novo modelo obteve uma melhora de $69,02 \%$ no valor do coeficiente linear quando comparado ao modelo global, além de coeficientes ângular e de correlação satisfatórios.

A Tabela 2 nos informa os valores das figuras de mérito para o modelo sendo que, comparado ao modelo global, o iPLS40 apresenta uma melhora de $85,72 \%$ na Sensibilidade e a sua maior Sensibilidade Analítica (consequentimente menor valor $\mathrm{de} \gamma^{1}$ ), indicam a melhora em seu desempenho.

Os limites de Detecção e de Quantificação são menores que o primeiro ponto da curva, porém maiores que os obtidos pelo modelo global. Tal fato pode ser justificado pela redução na disperção das amostras de referência, causada pela uniformidade nos valores de absorbância na faixa selecionada para construção do modelo iPLS40. Essa redução acarreta a um menor desvio padão do sinal e consequentimente a uma elevação nos valores de LD e LQ calculados.

\section{Comparação entre modelos}

O modelo gerado por iPLS foi comparado ao modelo global 


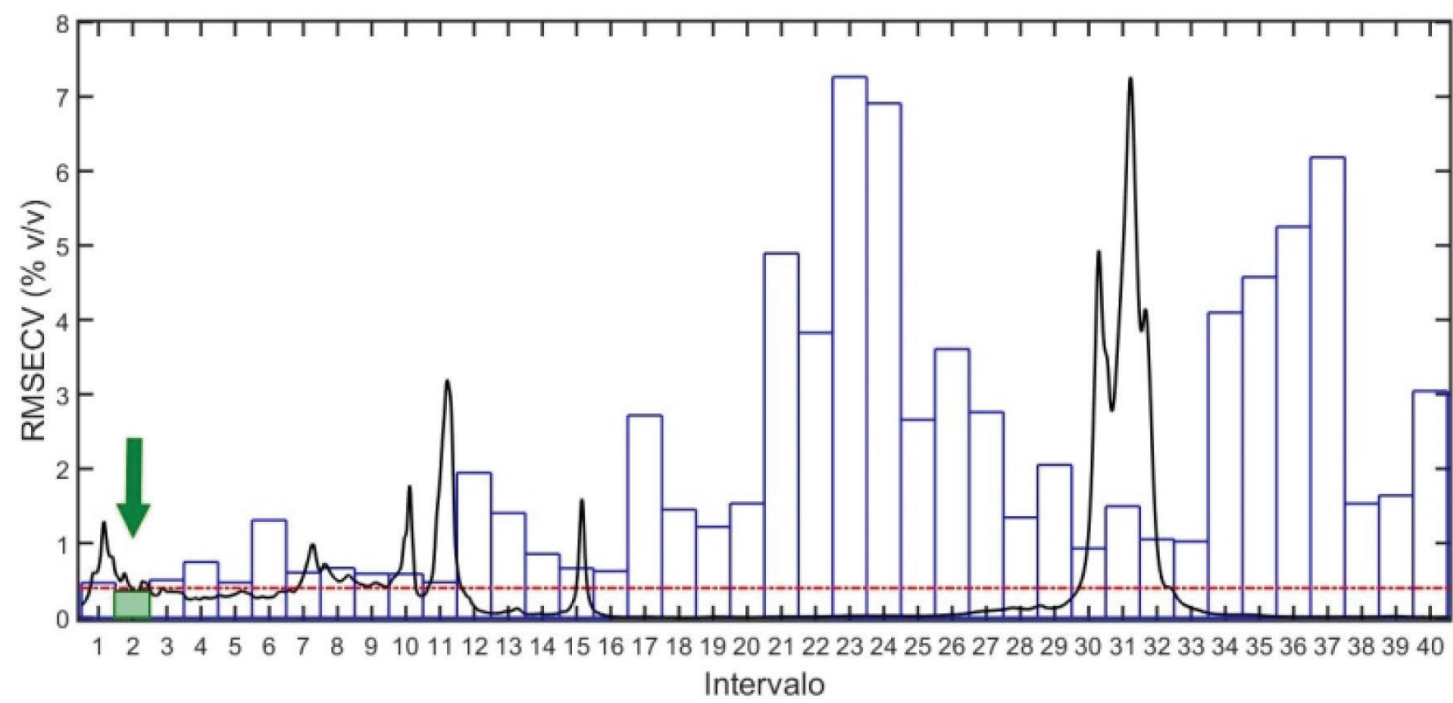

Figura 3. Gráfico iPLS4O

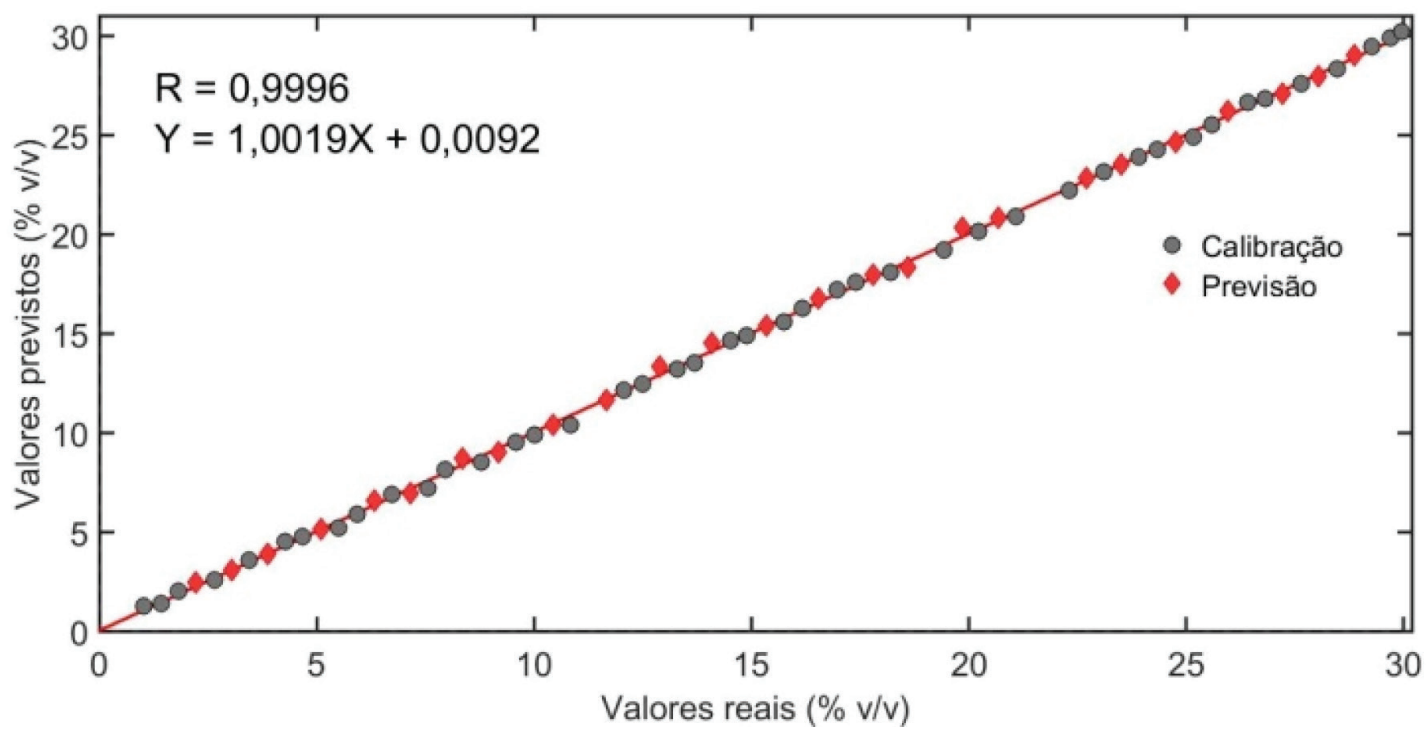

Figura 4. Regressão obtida após o uso do iPLS

Tabela 2. Figuras de mérito do modelo iPLS40 de biodiesel metílico de crambe-diesel

\begin{tabular}{ll}
\hline Sensibilidade (\% v/v) & 0,01 \\
Sensibilidade Analítica (\% v/v) & 30,9 \\
Inverso da Sensibilidade Analítica (\% v/v) & 0,03 \\
LQ $(\%$ v/v) & 0,27 \\
LD $(\%$ v/v) & 0,08 \\
\hline
\end{tabular}

através da estatística $\mathrm{F}$ conforme a Tabela 3, apresentando melhor desempenho de sua capacidade em prever as concentrações de biodiesel metílico de crambe presentes em misturas com diesel, como indicado pelo valor de $\mathrm{F}$ calculado maior que o $\mathrm{F}$ tabelado.

Juntamente com a melhora do RMSEP, o modelo iPLS4O obteve um RMSEC menor que o modelo global, contribindo para o aumento de sua Exatidão. A Sensibilidade e Sensibilidade Analítica aumentaram, tornando possível a distinção entre as amostras com concentrações divergentes em 0,03 (\% v/v). Todos esses fatores corroboram para a melhora da capacidade preditiva do modelo, viabilizando seu uso.

\section{CONCLUSÃO}

O método desenvolvido usando a técnica de espectroscopia MIR aliada à análise por Quadrados Mínimos Parciais se mostrou eficiente para a quantificação dos teores de biodiesel metílico de crambe em misturas com diesel, na faixa de concentração entre 1,00 a 30,00 (\% v/v). Vantagens como não usar solventes na preparação das amostras, construir uma única curva de calibração contemplando a mesma faixa

Tabela 3. Resultados obtidos por $i P L S$

\begin{tabular}{lccccc}
\hline MODELO & VARIÁVEIS & FAIXA & VL's & RMSEP $(\%$ v/v) & TESTE F* \\
\hline iPLS40 & 74 & $75: 148$ & 4 & 0,26 & 2,58 \\
GLOBAL & 2925 & $1: 2925$ & 4 & 0,41 & ---- \\
\hline
\end{tabular}

*Em relação ao modelo global $\left(\mathrm{F}_{(0,5 ; 25 ; 25)}=1,95\right)$. 
das duas curvas do método oficial e diminuição significativa da quantidade de pontos usados na construção do modelo, foram alcançadas. Assim o tempo necessário para a análise do teor dos biodieseis em misturas com diesel se torna menor e não há a geração de resíduos.

O modelo gerado após o uso do iPLS utilizou 74 variáveis para a construção da curva de calibração. Essa redução nas variáveis acarretou na melhora da capacidade preditiva e, além de diminuir a capacidade computacional necessária para o emprego dos modelos, propicia a construção de detectores para trabalhar na linha de produção, capturando a absorbância dos combustíveis dentro dos dutos, quantificando em tempo real seus teores de biodiesel.

\section{REFERÊNCIAS}

1. Andrade, D. C.; Mendonça, T. G. de; Romeiro, A. R.; Revibec 2018, 28, 66.

2. Nazareno, J. C.; Vieira, G. E. G.; Oliveira, G. G.; Bioenerg. Rev.: Diálogos 2015, 5, 8 .

3. Silveira, E. V.; Vilela, L. S.; Castro, C. F. S.; Lião, L. M.; Gambarra Neto, F. F.; Oliveira, P. S. M.; Ind. Crops Prod. 2017, 97, 545.

4. Salvador, P. M.; Martins, C. X.; Ferreira, L. F. R.; Américo, J. H. P.; Torres, N. H.; Bortoleto, G. G.; Bioenerg. Rev.: Diálogos 2016, 6, 31.

5. Vieira, J. S.; Sousa, T.; Rosas, L.; Lima, A.; Ronconi, C.; Mota, C.; Quim. Nova 2018, 41, 10.

6. Sustaita-Rodríguez, A.; Ramos-Sánchez, V. H.; Camacho-Dávila, A. A.; Zaragoza-Galán, G.; Espinoza-Hicks, J. C.; Chávez-Flores, D.; Chem. Cent. J. 2018, 12, 39.

7. Lalas, S.; Gortzi, O.; Athanasiadis, V.; Dourtoglou, E.; Dourtoglou, V.; J. Am. Oil Chem. 2012, 89, 2253.

8. Santos, K. A.; Bariccatti, R. A.; Cardozo-Filho, L.; Schneider, R.; Palú, F.; Silva, C.; Silva, E. A. da.; J. Supercrit. Fluids 2015, 104, 54.

9. Tavares, G. R.; Massa, T. B.; Gonçalves, J. E.; Silva, C.; Santos, W. D.; Renewable Energy 2017, 111, 659.

10. Brasil, R. F. Lei $N^{\circ}$. 13.263, de 23 de Março de 2016. Altera a Lei No 13.033, de 24 de Setembro de 2014, Para Dispor Sobre Os Percentuais de Adição de Biodiesel Ao Óleo Diesel Comercializado No Território Nacional. Diário Oficial da União 2016, 153. 93.
11. Aquino, G. S.; Hata, F. T.; Michelon, T. A.; Diniz, T. G.; Nicio, T. T.; Oliveira, A. L. M.; Watanabe, V. S.; Ventura, M. U.; Alexandrino, R. P.; Pescador, P. G. A.; Ind. Crops Prod. 2018, 121, 277.

12. http://www.epe.gov.br, accessada em maio 2020.

13. http://www.anp.gov.br, accessada em maio 2020.

14. Nabi, M. N.; Rasul, M. G. Energy Convers. Manage. 2018, 169, 326.

15. Guimarães, E.; Santana, F. B.; Gontijo, L. C.; Mitsutake, H.; Santos, D. Q.; Borges Neto, W.; Rev. Virtual Quim. 2015, 7, 2242.

16. ABNT.; ABNT NBR 15568 2008, 1.

17. Barros Neto, B.; Scarminio, I. S.; Bruns, R. E.; Quim. Nova 2006, 29, 1401.

18. Bruns, R. E.; Faigle, J. F. G.; Quim. Nova 1984, nr. abril, 84.

19. Beebe, K. R.; Kowalski, B. R.; Anal. Chem. 1987, 59, 1007A.

20. Massart, D. L.; Buydens, L. M. C.; Jing, S.; Lewi, P. J.; SmeyersVerbeke, J.; Handbook of Chemometric and Qualitimetrics, Part B; Elsevier: Amsterdam, 1998.

21. Otto, M.; Chemometrics: Statistics and Computer Application in Anal. Chem., $3^{\text {rd }}$ ed.; Gmbh, W. V., eds.; John Wiley \& Sons: Weinheim, Germany, 2016.

22. Andersen, C. M.; Bro, R.; J. Chemom. 2010, 24, 728.

23. Nørgaard, L.; Saudland, A.; Wagner, J.; Nielsen, J. P.; Munck, L.; Engelsen, S. B.; Appl. Spectrosc. 2000, 54, 413.

24. Lorber, A.; Faber, K.; Kowalski, B. R.; Anal. Chem. 1997, 69, 1620.

25. Lorber, A.; Anal. Chem. 1986, 58, 1167.

26. Valderrama, P.; Braga, J. W. B.; Poppi, R. J.; Quim. Nova 2009, 32, 1278.

27. Filgueiras, P. R.; Alves, J. C. L.; Poppi, R. J.; Talanta 2014, 119, 582.

28. Martens, H.; Naes, T.; Multivariate Calibration, John \& Sons: New York, 1989.

29. Valderrama, P.; Braga, J. W. B.; Poppi, R. J.; J. Agric. Food Chem. 2007, 55,8331 .

30. Sitoe, B. V.; Máquina, A. D. V.; Gontijo, L. C.; Oliveira, L. R.; Santos, D. Q.; Borges Neto, W.; Anal. Lett. 2020, 53, 589.

31. ASTM E1655-05 Standard; ASTM International 2012, 05, 29. 